\title{
MiniDMAIC: Uma Abordagem para Análise e Resolução de Causas em Projetos de Desenvolvimento de Software
}

\author{
Carla Ilane M. Bezerra ${ }^{1,2}$, Ciro Carneiro Coelho ${ }^{2}$ \\ , Fca. Márcia G. S. Gonçalves ${ }^{1}$, Carlo Giovano S. Pires ${ }^{2}$, Gabriela Telles ${ }^{2}$, Adriano \\ Bessa Albuquerque ${ }^{1}$ \\ ${ }^{1}$ Mestrado em Informática Aplicada, Universidade de Fortaleza \\ Av. Washington Soares 1321, 60811-341 - Fortaleza - CE, Brasil \\ ${ }^{2}$ Instituto Atlântico, Rua Chico Lemos, 946, 60822-780 - Fortaleza - CE, Brasil \\ \{carlailane, cgiovano, gabi\}eatlantico.com.br, ccoelho@gmail.com, \\ marcia@hotmail.com, adriano.ba@terra.com.br.
}

Abstract: Handling problems and defects in software development projects is still a difficult matter in many organizations. The problems analysis, when performed, usually do not focus on the problem sources and root causes, leading to bad decisions that do not solve the problem. It is also hard for organizations to implement a Causal Analysis and Resolution (CAR) process in software projects, as described in the CMMI level 5 because projects usually have to deal with very limited resources. This paper presents the MiniDMAIC, an approach for defects and problems causal analysis and resolution based on Six Sigma's DMAIC methodology and the Causal Analysis and Resolution process area from CMMI.

Resumo: O tratamento dos problemas e defeitos encontrados nos projetos de software ainda é deficiente na maioria das organizações. As análises em geral não focam suficientemente no problema e suas possiveis origens, levando a tomadas decisões erradas que acabam por não resolver o problema. Também é dificil a implementação de um processo de Análise e Resolução de Causas (CAR) em projetos, conforme prescrito pelo nivel 5 do CMMI, devido aos recursos limitados com os quais os mesmos têm que trabalhar. Este trabalho propõe o MiniDMAIC, uma abordagem para análise e resolução de causas de defeitos $e$ problemas em projetos de desenvolvimento de software com base na metodologia DMAIC do Six Sigma e na área de processo de CAR do CMMI.

\section{Introdução}

A busca incessante por produtos e serviços cada vez melhores leva muitas organizações a pesquisar e implantar várias técnicas, ferramentas e normas capazes de alavancar a qualidade de seus produtos. Dentro do contexto da busca por excelência, surge como solução para diminuir custo e melhorar a qualidade a implementação de iniciativas tais como o CMMI (2006) e o Six Sigma [Tayntor 2003].

Um dos aspectos fundamentais da melhoria da qualidade é a análise e resolução de problemas. Para isso, pode ser utilizado um método formal de resolução de problemas, que pode trazer inúmeros benefícios, tais como [Banas Qualidade 2007]:

- Evitar que os solucionadores de problemas passem direto para a conclusão;

- Garantir a análise da causa-raiz; 
- Desmistificar o processo de solução de problemas;

- Estabelecer ferramentas analíticas a usar e determinar quando utilizá-las.

Nesse contexto, a utilização de ferramentas da metodologia Six Sigma, como o DMAIC, tem se destacado. Ao contrário de outras metodologias para resolução de problemas, que focam apenas na eliminação do problema em si, a metodologia DMAIC [Rath and Strong 2005] empregada pelo Six Sigma abrange desde a seleção dos problemas que merecem um tratamento mais aprofundado até o controle dos resultados obtidos no decorrer do tempo.

A metodologia DMAIC mostra passo a passo como tais problemas devem ser tratados, agrupando as principais ferramentas da qualidade, ao mesmo tempo em que cria uma rotina padronizada na solução de problemas com uma comprovada eficiência na aplicação em organizações de software.

Apesar de adequados para o nível organizacional, os métodos formais de resolução de problemas podem ser inviáveis no nível projetos. Um dos grandes desafios enfrentados por empresas que almejam o nível 5 do CMMI é justamente a implantação da área de processo de Análise e Resolução de Causas (Causal Analysis and Resolution - CAR) no contexto de projetos de software, uma vez que estes geralmente possuem recursos bastante limitados. Com isso, são tomadas apenas ações imediatas para resolução dos problemas e, na maioria das vezes, os mesmos voltam a acontecer.

Apesar de todos os benefícios do uso da metodologia Six Sigma em conjunto com o CMMI, a implementação da área de processo Análise e Resolução de Causas em projetos de software muitas vezes torna-se inviável, pelos seguintes motivos:

- Projetos DMAIC têm duração entre 3 a 6 meses. No entanto, projetos requerem rapidez na resolução de seus problemas, não podendo esperar muito tempo;

- Devido à grande necessidade da utilização de ferramentas estatísticas, o DMAIC pode tornar-se excessivamente caro; a economia obtida pode ser menor do que o custo para alcançar melhorias, e os projetos geralmente possuem recursos limitados.

- O nível de qualificação da equipe DMAIC é bastante rigoroso; no entanto, no contexto de projetos de desenvolvimento de software, outros atributos como domínio do negócio e de gerência de projetos podem trazer maiores resultados do que o fato de ter uma equipe com grande conhecimento em estatística.

Diante deste contexto, este trabalho visa à elaboração de uma metodologia baseada no DMAIC do Six Sigma, denominada MiniDMAIC, para tratar a área de processo de Análise e Resolução de Causas do CMMI em projetos de desenvolvimento de software, procurando reduzir as desvantagens acima descritas de uso do DMAIC. Também visa mostrar a aplicação da metodologia em projetos de desenvolvimento de software em uma organização a partir de uma ferramenta workflow, onde foi implementada as práticas do MiniDMAIC.

Este trabalho está organizado em cinco seções, além desta introdução. Na seção 2, apresentamos o embasamento teórico referente ao Six Sigma e, mais detalhadamente, à metodologia DMAIC. Na seção 3, abordamos o CMMI e a área de processo de Análise e Resolução de Causas pertencente ao nível 5 de maturidade. Na seção 4, apresentamos a abordagem proposta, denominada MiniDMAIC. Aspectos referentes à 
utilização do MiniDMAIC em projetos reais, bem como os resultados obtidos, são apresentados na seção 5. Por fim, na seção 6, apresentamos as considerações finais e limitações da metodologia proposta.

\section{O Six Sigma e a Metodologia DMAIC}

O Six Sigma é um método que se concentra na diminuição ou eliminação da incidência de erros, defeitos e falhas em um processo. A metodologia Six Sigma visa também reduzir a variabilidade do processo, podendo ser aplicada na maioria dos setores da atividade econômica [Smith 2000].

Alcançar o Six Sigma significa reduzir defeitos, erros e falhas ${ }^{1}$ a zero e atingir a quase perfeição no desempenho dos processos. A metodologia associa um rigoroso enfoque estatístico a um arsenal de ferramentas que são empregadas com o objetivo de caracterizar as fontes da variabilidade para demonstrar como esse conhecimento pode controlar e aperfeiçoar os resultados do processo [Watson 2001].

A metodologia Six Sigma procura definir as causas óbvias e não óbvias que afetam o processo de forma a eliminá-las, ou melhorá-las e controlá-las [Rotondaro 2002].

O Six Sigma apresenta algumas metodologias para o tratamento de problemas e melhorias, tais como DMAIC (Define, Measure, Analyse, Improve and Control), DCOV (Define, Caracterize, Optimize, Verify) e DFSS (Design For Six Sigma). Neste trabalho, será utilizada a metodologia DMAIC.

A metodologia DMAIC foi criada de forma mais padronizada pela General Electric e, de acordo com Tayntor (2003), é a mais utilizada nas empresas que implementam o Six Sigma, e também a mais indicada para o desenvolvimento de software.

A metodologia DMAIC é constituída por cinco fases: definir, medir, analisar, melhorar e controlar. Na fase "definir", é necessário identificar o problema e então definir as oportunidades existentes para solucioná-lo de acordo com os requisitos dos clientes. Na fase "medir", deve-se verificar a situação atual através de medições quantitativas do desempenho, para que as decisões posteriores sejam baseadas em fatos. $\mathrm{Na}$ fase "analisar", deve-se determinar as causas do desempenho encontrado e analisar as oportunidades existentes. Após ter feito essa análise é possível perceber pontos para melhoria do desempenho, assim as melhorias podem ser implantadas na fase "melhorar". Na fase "controlar", deve-se assegurar a melhoria, através de um controle do desempenho do processo implantado.

Na Tabela 1 são descritos os objetivos das fases do modelo DMAIC de acordo com Tayntor (2003).

1 Na metodologia Six Sigma, os defeitos, os erros e as falhas são qualquer desvio de uma característica que gere insatisfação ao cliente [Blauth 2003]. 
Tabela 1. Objetivos definidos por Tayntor (2003) para o modelo DMAIC

\begin{tabular}{|c|c|}
\hline \multicolumn{2}{|r|}{ O Modelo DMAIC } \\
\hline Fase & Objetivos \\
\hline 气 & $\begin{array}{l}\text { Identificar o problema e os clientes; definir e priorizar os requisitos do cliente; definir o } \\
\text { processo atual. }\end{array}$ \\
\hline$\stackrel{\Xi}{\Xi}$ & $\begin{array}{l}\text { Confirmar e quantificar o problema; medir as diversas etapas do processo atual; rever e } \\
\text { esclarecer o escopo do problema, se necessário; definir os resultados desejados }\end{array}$ \\
\hline 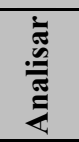 & Determinar a causa raiz do problema; propor soluções \\
\hline 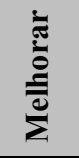 & Priorizar soluções; desenvolver e implementar as soluções que trarão maiores benefícios \\
\hline 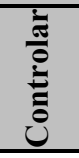 & $\begin{array}{l}\text { Medir as melhorias; comunicar e comemorar o sucesso; assegurar que as melhorias no processo } \\
\text { serão implantadas }\end{array}$ \\
\hline
\end{tabular}

Pande (2001) ressalta que não se pode utilizar o DMAIC para qualquer melhoria. Um projeto de melhoria Six Sigma, segundo o autor, deve ter três qualificações:

- Há uma lacuna entre o desempenho atual e o desempenho necessário/desejado;

- A causa do problema não é claramente compreendida;

- A solução não é predeterminada, nem é a solução ótima aparente.

Além disso, devem ser observados os critérios de viabilidade, tais como: os recursos necessários, a habilidade disponível, a complexidade, a probabilidade de sucesso e o apoio ou engajamento da equipe.

\section{O CMMI e a Análise e Resolução de Causas}

O Capability Maturity Model Integration (CMMI) [Chrissis 2006] é um modelo de maturidade de desenvolvimento de produtos desenvolvido pelo Software Engineering Institute (SEI), que está cada vez mais sendo adotado nas empresas, uma vez que esse modelo busca orientar as organizações na implementação de melhorias contínuas em seu processo de desenvolvimento.

\subsection{O Nível 5 de Maturidade}

O foco do nível 5 de maturidade está na melhoria contínua dos processos. Enquanto o nível 4 foca nas causas especiais de variação no processo da organização, o nível 5 tenta encontrar as causas comuns e tratá-las, resultando em inúmeras melhorias, que são introduzidas de forma disciplinada. Medições são usadas para selecionar as melhorias e estimar os custos e benefícios em atender as melhorias propostas. As mesmas medições podem ser usadas para justificar esforços para futuras melhorias [Kulpa 2003].

O nível 5 do CMMI é composto por duas áreas de processo: Inovação e Desenvolvimento Organizacional (Organizational Innovation and Deployment - OID) e 
Análise e Resolução de Causas (Causal Analysis and Resolution - CAR), sendo esta última o foco deste trabalho.

O objetivo da PA Análise e Resolução de Causas (Causal Analysis and Resolution - CAR) é identificar causas de defeitos e outros problemas e tomar ações para prevenir a ocorrência deles no futuro.

A Tabela 2 apresenta o relacionamento dos objetivos específicos (SG) com suas respectivas práticas específicas (SP) para essa área de processo.

Tabela 2. Análise e Resolução de Causas no CMMI [Chrissis 2006]

\begin{tabular}{|l|l|l|}
\hline SG 1 & \multicolumn{2}{|l|}{ Determinar as Causas dos Defeitos } \\
\hline & SP 1.1 & Selecionar os dados para análise dos defeitos \\
\cline { 2 - 3 } & SP 1.2 & Analisar as causas \\
\hline SG 2 & Tratar as Causas dos Defeitos \\
\hline & SP 2.1 & Implementar as ações propostas \\
\cline { 2 - 3 } & SP 2.2 & Avaliar os efeitos das mudanças \\
\cline { 2 - 3 } & SP 2.3 & Registrar os dados \\
\hline
\end{tabular}

\section{MiniDMAIC}

O MiniDMAIC é uma estratégia que visa à simplificação do modelo DMAIC com o intuito de tratar as causas e resolução de problemas em projetos de desenvolvimento de software de forma mais prática, rápida, com menos riscos e custos, prevenindo recorrências futuras, implantando melhorias no processo de desenvolvimento e assim, aumentando cada vez mais a satisfação dos seus clientes.

As principais características do MiniDMAIC são:

- Curta duração (variando de 1 a 6 semanas);

- Requer conhecimento básico de estatística;

- Associada a riscos;

- Custo baixo;

- Específico para projetos de desenvolvimento de software.

Os problemas que merecem um tratamento mais criterioso através da aplicação da metodologia MiniDMAIC podem ser definidos a nível organizacional (Ex.: limites de controle, quantidade de defeitos, etc.) e serem refinados durante o planejamento do projeto. Deve estar bem claro para a equipe do projeto a diferença entre os problemas que requerem apenas ações simples e imediatas, e os problemas que requerem o tratamento definido na metodologia MiniDMAIC.

Exemplos de problemas de projetos que merecem o tratamento através da abordagem MiniDMAIC são:

- Projeto fora de controle, podendo ser considerados indicadores críticos para o cliente e desvios das metas organizacionais (Ex.: produtividade, desvio na entrega, densidade de defeitos, etc.)

- Problemas cuja causa raiz seja duvidosa;

- Problemas relacionados a critérios de aceitação do projeto pelo cliente.

Como apoio à metodologia, podem ser utilizadas ferramentas como planilhas eletrônicas, ferramentas de gestão de projetos, entre outras. 


\subsection{A Fase "Definir"}

A fase definir consiste na definição do problema, fontes e metas, além da formação da equipe, conforme podemos ver na Tabela 3.

\section{Tabela 3. Passos da fase "Definir"}

\begin{tabular}{|c|c|c|}
\hline \multirow{4}{*}{ : } & $\begin{array}{c}\text { Passo } 1 \text { - Definir } \\
\text { o Problema }\end{array}$ & $\begin{array}{l}\text { Deve ser definido o problema que será tratado para que esteja clara a sua } \\
\text { importância e sejam definidos os seus objetivos. É importante descrever qual o } \\
\text { impacto ou conseqüências do problema sobre o projeto. Esta descrição deve } \\
\text { estar focando somente nos sintomas e não em causas ou soluções. }\end{array}$ \\
\hline & $\begin{array}{l}\text { Passo } 2 \text { - } \\
\text { Determinar a } \\
\text { Fonte do } \\
\text { Problema }\end{array}$ & $\begin{array}{l}\text { Esta etapa deve mostrar qual foi a fonte que mostrou a ocorrência do } \\
\text { problema. Exemplos de fontes de problemas em projetos de desenvolvimento } \\
\text { de software são: } \\
\text { - Resultados de pesquisas de satisfação com o cliente; } \\
\text { - Reclamações do cliente; } \\
\text { - Indicadores do projeto; } \\
\text { - Resultados de estudos de benchmarking; } \\
\text { - Resultados de avaliações da qualidade. }\end{array}$ \\
\hline & $\begin{array}{c}\text { Passo } 3 \text { - Definir } \\
\text { as Metas }\end{array}$ & $\begin{array}{l}\text { Nesta etapa são definidas as metas que devem ser atingidas com a execução da } \\
\text { metodologia MiniDMAIC para tratamento do problema em questão. As metas } \\
\text { devem ser definidas de forma quantitativa, podendo ser através de alguma } \\
\text { medição que define o problema a ser atacado e também do cálculo do retorno } \\
\text { de investimento (ROI - return of investment) planejado. }\end{array}$ \\
\hline & $\begin{array}{c}\text { Passo } 4 \text { - Formar } \\
\text { a Equipe }\end{array}$ & $\begin{array}{l}\text { Em uma ação MiniDMAIC não há necessidade de se ter Black } \text { Belts }^{2} \text { na } \\
\text { liderança. Por serem simples e diretamente relacionadas ao projeto, é } \\
\text { necessário apenas um conhecimento básico sobre Six Sigma e treinamento na } \\
\text { metodologia MiniDMAIC. O mais relevante é o entendimento relacionado ao } \\
\text { negócio do projeto e técnicas de gestão, sendo, portanto, indicado que o } \\
\text { Gerente do Projeto lidere o MiniDMAIC. O tamanho da equipe MiniDMAIC } \\
\text { pode variar de acordo com a necessidade do problema. Em algumas situações, } \\
\text { podemos ter apenas o gerente do projeto e um membro da equipe contando } \\
\text { com a participação de outras pessoas apenas em determinados passos, como } \\
\text { apoio de um líder Green Belt }{ }^{3} \text { (principalmente na fase medir e analisar). }\end{array}$ \\
\hline
\end{tabular}

\subsection{A Fase "Medir"}

A fase medir consiste na análise das medições (existentes ou a definir) relacionadas ao problema, bem como o cálculo do nível sigma atual, conforme podemos ver na Tabela 4.

2 Black Belts: Colaboradores que trabalham aplicando os conceitos e as ferramentas do Six Sigma em projetos. Sua formação depende de um treinamento intensivo em estatística e em técnicas para solução de problemas. Eles normalmente atuam identificando em diferentes processos, oportunidades a serem exploradas por meio de projetos [Harry e Schroeder, 2000].

3 Green Belts: Colaboradores em diferentes partes da organização que aplicam o Six Sigma no seu dia-adia. Sua formação envolve um treinamento menos intensivo que o dos Black Belts [Harry e Schroeder, 2000]. 
Tabela 4. Passos da fase "Medir"

\begin{tabular}{|c|c|l|}
\hline \multirow{2}{*}{$\begin{array}{c}\text { Passo 1 - Planejar } \\
\text { Medições }\end{array}$} & $\begin{array}{l}\text { Nesta etapa deve ser analisado se existe a necessidade de uma nova medição } \\
\text { que forneça maiores evidências para o problema em questão. Em grande parte } \\
\text { das situações, as medições já estarão sendo conduzidas de acordo com o } \\
\text { processo definido que atende à PA de Medição e Análise (Measurement and } \\
\text { Analysis - MA). }\end{array}$ \\
\hline $\begin{array}{c}\text { Passo 2 - Medir } \\
\text { Situação Atual }\end{array}$ & $\begin{array}{l}\text { As medições selecionadas no passo anterior devem ser utilizadas para medir a } \\
\text { situação atual do projeto. Posteriormente, essas mesmas medições serão } \\
\text { utilizadas para aferir a melhoria obtida. }\end{array}$ \\
\hline
\end{tabular}

\subsection{A Fase "Analisar"}

A fase analisar consiste na definição da categoria do problema, determinação de suas causas e possíveis ações para tratá-las, e, avaliação dos riscos associados às causas raiz do problema, conforme podemos ver na Tabela 5 .

\section{Tabela 5. Passos da fase "Analisar"}

\begin{tabular}{|c|c|c|}
\hline \multirow[t]{2}{*}{ 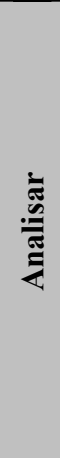 } & $\begin{array}{l}\text { Passo } 1 \text { - Determinar } \\
\text { as Causas do } \\
\text { Problema }\end{array}$ & $\begin{array}{l}\text { Este é um dos passos mais importantes do MiniDMAIC, uma vez que seu } \\
\text { propósito é encontrar a causa raiz do problema. Caso esta atividade não seja } \\
\text { feita corretamente, o resultado do MiniDMAIC pode ser comprometido, pois } \\
\text { todas as atividades seguintes terão como base o resultado deste passo. Sendo } \\
\text { assim, é importante que participem desta etapa as pessoas que possuem } \\
\text { conhecimento a respeito do problema e que possam contribuir com } \\
\text { informações sobre suas causas. Exemplos de técnicas para determinar as } \\
\text { causas do problema são: brainstorming, cinco porquês, diagrama de causa e } \\
\text { efeito [Ishikawa, 1998], gráfico de pareto [Juran,1991], entre outros. }\end{array}$ \\
\hline & $\begin{array}{l}\text { Passo } 2 \text { - Definir } \\
\text { Ações Candidatas }\end{array}$ & $\begin{array}{l}\text { Neste passo devem ser levantadas as possíveis ações que venham a tratar o } \\
\text { problema em questão. Este passo será complementado na próxima fase onde } \\
\text { as ações serão priorizadas. }\end{array}$ \\
\hline
\end{tabular}

\subsection{A Fase "Melhorar"}

A fase melhorar consiste na priorização e aprovação das ações propostas, elaboração e execução do plano de ação, bem como o acompanhamento dos resultados obtidos, conforme podemos ver na Tabela 6 .

Tabela 6. Passos da fase "Melhorar"

\begin{tabular}{|c|c|c|}
\hline \multirow{3}{*}{ 竞 } & $\begin{array}{l}\text { Passo } 1 \text { - Priorizar } \\
\text { as Ações } \\
\text { Propostas }\end{array}$ & $\begin{array}{l}\text { A priorização das ações propostas deve ser feita de acordo com o processo } \\
\text { definido para a área de Análise e Tomada de Decisão (Decision Analysis and } \\
\text { Resolution - DAR), que define as diretrizes para seleção das soluções } \\
\text { propostas. Caso seja identificada apenas uma ação para tratamento do } \\
\text { problema, deve ser feita uma análise de viabilidade de execução da mesma. }\end{array}$ \\
\hline & $\begin{array}{l}\text { Passo } 2 \text { - Obter } \\
\text { Aprovação }\end{array}$ & $\begin{array}{l}\text { Deve ser realizada uma avaliação de impacto referente à implementação das } \\
\text { ações priorizadas, resumindo seus efeitos positivos e negativos. Essas } \\
\text { informações devem ser submetidas à aprovação da gerência sênior. }\end{array}$ \\
\hline & $\begin{array}{l}\text { Passo } 3 \text { - Elaborar } \\
\text { e Executar Plano } \\
\text { de Ação }\end{array}$ & $\begin{array}{l}\text { Um plano de ação para execução das ações priorizadas e aprovadas deve ser } \\
\text { elaborado. Este plano deve conter as seguintes informações: } \\
\text { - Tarefas a serem executadas; } \\
\text { - Responsável pela execução da tarefa; } \\
\text { - Esforço necessário para execução da tarefa; } \\
\text { - Prazo para conclusão da tarefa. } \\
\text { A execução do plano de ação se dá através da implementação das suas } \\
\text { tarefas. }\end{array}$ \\
\hline
\end{tabular}


Passo 4 -

Acompanhar Ações
Neste passo as tarefas devem ser monitoradas para que se tenha uma visibilidade do avanço do MiniDMAIC. Estes resultados devem ser acompanhados de acordo com o processo de Monitoramento e Controle do Projeto (Project Monitoring and Control - PMC).

\subsection{A Fase "Controlar"}

A fase controlar consiste na avaliação dos resultados obtidos e divulgação dos resultados e lições aprendidas obtidos, conforme podemos ver na Tabela 7.

\section{Tabela 7. Passos da fase "Controlar"}

\begin{tabular}{|c|c|c|}
\hline & $\begin{array}{l}\text { Passo } 1 \text { - Avaliar } \\
\text { Resultados }\end{array}$ & $\begin{array}{l}\text { Na avaliação dos resultados obtidos deve ser feita uma análise se a meta } \\
\text { estabelecida na fase "Definir" foi atingida, bem como uma análise do custo- } \\
\text { benefício do tratamento do problema utilizando a metodologia MiniDMAIC. } \\
\text { O custo benefício do projeto pode ser medido através do cálculo do retorno } \\
\text { de investimento (ROI - return of investment). }\end{array}$ \\
\hline 离 & $\begin{array}{l}\text { Passo } 2 \text { - Divulgar } \\
\text { os Principais } \\
\text { Resultados e } \\
\text { Lições Aprendidas }\end{array}$ & $\begin{array}{l}\text { Ao final da execução do MiniDMAIC os resultados obtidos devem ser } \\
\text { compartilhados com toda a organização através de um repositório } \\
\text { organizacional acessível a todos os projetos. O compartilhamento destas } \\
\text { informações pode servir de insumo para o tratamento de problemas similares } \\
\text { em outros projetos. A forma de divulgação deve seguir o processo definido } \\
\text { para a PA de Foco no Processo Organizacional (Organizational Processos } \\
\text { Focus - OPF), que define como serão compartilhadas as lições aprendidas } \\
\text { pela organização. Caso sejam identificadas possíveis melhorias nos processos } \\
\text { organizacionais, as mesmas devem ser encaminhadas para o Grupo de } \\
\text { Engenharia de Processos (Engineering Process Group - EPG) para que } \\
\text { possam se analisadas e devidamente tratadas. }\end{array}$ \\
\hline
\end{tabular}

\subsection{MiniDMAIC x DMAIC}

A abordagem proposta tem como base os passos definidos por Tayntor (2003) para o modelo DMAIC no âmbito do desenvolvimento de software. Alguns passos foram suprimidos da metodologia e outros foram adaptados para tornar mais fácil a execução do processo nos projetos de desenvolvimento de software. Foi tomado o cuidado para que a essência do modelo não fosse alterada, conforme os objetivos que Tayntor (2003) define na Tabela 1.

A grande diferença entre o MiniDMAIC e o DMAIC é que o DMAIC através de uma análise e resolução das causas para o problema definido tem como objetivo maior a melhoria de um processo que já existe e é executado na organização. O MiniDMAIC apenas trata as causas a nível do projeto e tem como objetivo a prevenção e tratamento dos problemas definidos através da análise e resolução da(s) causa(s) raiz(es) destes problemas, não modificando necessariamente o processo organizacional.

Além disso, o DMAIC exige a comprovação estatística das causas dos problemas e das melhorias alcançadas, o que não é necessário no MiniDMAIC, que levanta e prioriza as causas por meio de ferramentas mais simples, como Diagrama de Ishikawa e Gráfico de Pareto, e analisa as melhorias obtidas observando o progresso dos indicadores do projeto.

\subsection{MiniDMAIC x CAR}

Para um melhor entendimento do relacionamento do MiniDMAIC com a PA de Análise e Resolução de Causas (CAR), foi realizado um mapeamento entre os passos do 
MiniDMAIC com as práticas específicas de CAR como podemos ver na Tabela 8. Vale ressaltar que tal relacionamento não implica em estar cobrindo toda a prática, uma vez que a mesma não está atrelada apenas a projetos, mas também possui subpráticas no nível organizacional.

Tabela 8. Relacionamento dos Passos do MiniDMAIC com as Práticas Específicas de CAR

\begin{tabular}{|c|c|c|c|}
\hline Fase & MiniDMAIC (Passos) & $\begin{array}{c}\text { CAR (Práticas } \\
\text { Específicas) }\end{array}$ & Observações \\
\hline \multirow{4}{*}{ : } & Passo 1 - Definir o Problema & 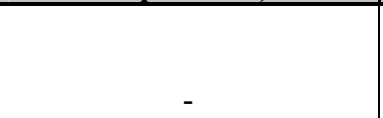 & $\begin{array}{l}\text { Relacionado à PA } \\
\text { Quantitative Project } \\
\text { Management - QPM }\end{array}$ \\
\hline & $\begin{array}{l}\text { Passo } 2 \text { - Determinar a Fonte do } \\
\text { Problema }\end{array}$ & - & \\
\hline & Passo 3 - Definir as Metas & - & $\begin{array}{l}\text { Relacionado à PA } \\
\text { Quantitative Project } \\
\text { Management - QPM }\end{array}$ \\
\hline & Passo 4 - Formar a Equipe & - & $\begin{array}{l}\text { Relacionado à GP } 2.7 \text { - } \\
\text { Identificar e Envolver os } \\
\text { Stakeholders Relevantes }\end{array}$ \\
\hline \multirow{2}{*}{$\sum_{\Sigma}^{\vdots}$} & Passo 1 - Planejar Medições & $\begin{array}{l}\text { SP } 1.1 \text { - Selecionar os } \\
\text { dados de defeitos para } \\
\text { análise }\end{array}$ & \\
\hline & Passo 2 - Medir Situação Atual & $\begin{array}{l}\text { SP } 1.1 \text { - Selecionar os } \\
\text { dados de defeitos para } \\
\text { análise }\end{array}$ & \\
\hline \multirow{2}{*}{ 离 } & $\begin{array}{l}\text { Passo } 1 \text { - Determinar as Causas do } \\
\text { Problema }\end{array}$ & SP 1.2 - Analisar as Causas & \\
\hline & $\begin{array}{l}\text { Passo } 2 \text { - Definir Ações } \\
\text { Candidatas }\end{array}$ & SP 1.2 - Analisar as Causas & \\
\hline \multirow{5}{*}{ 离 } & \multirow{2}{*}{$\begin{array}{l}\text { Passo } 1 \text { - Priorizar as Ações } \\
\text { Propostas }\end{array}$} & SP 1.2 - Analisar as Causas & \\
\hline & & $\begin{array}{l}\text { SP } 2.1 \text { - Implementar as } \\
\text { Ações Propostas }\end{array}$ & \\
\hline & Passo 2 - Obter Aprovação & ]$_{3}$ & $\begin{array}{l}\text { Relacionado à GP } 2.10 \text { - } \\
\text { Revisar o Status com o } \\
\text { Gerente de Alto Nível }\end{array}$ \\
\hline & $\begin{array}{l}\text { Passo } 3 \text { - Elaborar e Executar Plano } \\
\text { de Ação }\end{array}$ & $\begin{array}{l}\text { SP } 2.1 \text { - Implementar as } \\
\text { Ações Propostas }\end{array}$ & \\
\hline & Passo 4 - Acompanhar Ações & 3 & $\begin{array}{l}\text { Relacionado à PA Project } \\
\text { Monitoring and Control- } \\
\text { PMC }\end{array}$ \\
\hline \multirow{2}{*}{ 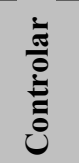 } & Passo 1 - Avaliar Resultados & $\begin{array}{l}\text { SP } 2.2 \text { - Avaliar os Efeitos } \\
\text { das Mudanças }\end{array}$ & \\
\hline & $\begin{array}{l}\text { Passo } 2 \text { - Divulgar os Principais } \\
\text { Resultados e Lições Aprendidas }\end{array}$ & $\begin{array}{l}\text { SP } 2.3 \text { - Registrar os } \\
\text { Dados }\end{array}$ & \\
\hline
\end{tabular}

\section{MiniDMAIC na Prática}

A metodologia MiniDMAIC foi aplicada no contexto de projetos do Instituto Atlântico para utilização de análise de causas e defeitos dentro dos projetos de desenvolvimento de software. O Instituto Atlântico é uma instituição de pesquisa e desenvolvimento de médio porte localizada em Fortaleza-Ceará e possui nível 3 de maturidade no modelo CMMI-SW e norma ISO 9001:2000. Visando a melhoria contínua dos seus processos, o 
Atlântico encontra-se em processo de implantação dos níveis 4 e 5 de maturidade do modelo CMMI com o apoio da metodologia Six Sigma.

Para disparar um MiniDMAIC dentro dos projetos da organização foram adotados os seguintes critérios:

- Defeitos recorrentes;

- Número elevado de defeitos em revisões técnicas (quando a causa é desconhecida);

- Número elevado de defeitos classificados como critical e blocker em testes sistêmicos (quando a causa é desconhecida);

- Número elevado de defeitos encontrados pelo cliente (quando a causa é desconhecida);

- Projeto fora dos limites de especificação das baselines;

- Projeto fora dos limites de controle das baselines.

Todos os passos do MiniDMAIC foram implementados na ferramenta Jira ${ }^{4}$, uma ferramenta comercial de gerenciamento de workflow que pode ser facilmente customizada. Segue abaixo, na Figura 1, um exemplo da tela de criação de uma ação MiniDMAIC no Jira.

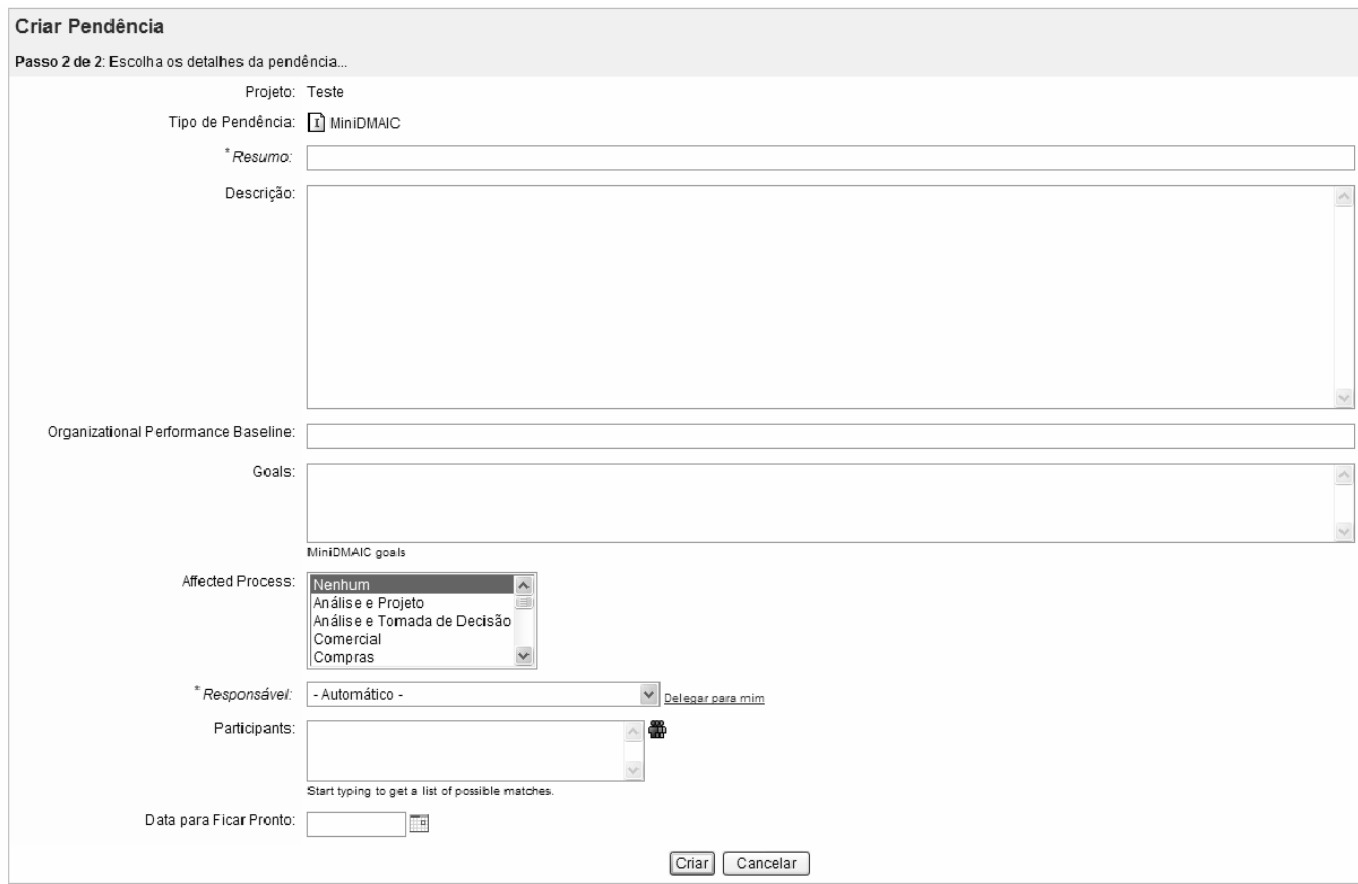

Figura 1. Tela do Jira implementando o MiniDMAIC

Na Figura 1 podemos observar as informações necessárias para que seja dado início a um MiniDMAIC:

4 Site do Jira: www.atlassian.com/software/jira/ 
- Project: este campo apresenta o nome do projeto e é preenchido automaticamente, de acordo com o projeto em questão;

- Issue Type: para iniciar uma ação MiniDMAIC, deve ser selecionada a opção "MiniDMAIC";

- Summary: este campo deve conter o problema a ser tratado.

- Description: este campo deve conter uma descrição detalhada do problema, citando desde quando o mesmo existe e qual o seu impacto ou conseqüências sobre o projeto. Esta descrição deve estar focando somente nos sintomas e não em causas ou soluções;

- Organizational Performance Baseline: este campo deve mostrar qual é a baseline de desempenho organizacional relacionada ao problema que está sendo tratado. A baseline de desempenho organizacional servirá de parâmetro para as metas de melhoria que serão estabelecidas posteriormente;

- Goals: este campo deverá conter a metas e objetivos a serem atingidos com a execução da ação MiniDMAIC. Estas metas devem ser quantificáveis e viáveis;

- Affected Process: neste campo devem ser selecionados os processos que serão afetados com a execução da ação MiniDMAIC. Podem ser selecionados um ou mais processos;

- Assign To: este campo deve conter o nome do responsável que estará conduzindo a ação MiniDMAIC. Esta função geralmente será conduzida pelo Coordenador do Projeto. Caso seja julgado necessário, pode ser designado um colaborador com formação de Green Belt (o QA do projeto, por exemplo) para apoiar o Coordenador;

- Participants: este campo deve listar as pessoas que participarão da ação MiniDMAIC, além do responsável pela sua condução. Podem ser selecionadas pessoas da equipe do projeto que estejam relacionadas direta ou indiretamente no problema a ser tratado, além de pessoas de fora do projeto que detenham conhecimento e possam colaborar na condução da ação MiniDMAIC;

- Due Date: Este campo deve conter o prazo máximo para término da ação. Esse período geralmente varia de 1 a 6 semanas, de acordo com a complexidade do problema e o tempo necessário para se obter os resultados esperados.

Posteriormente, a ação MiniDMAIC será completada com outras informações, a medida que as fases do MiniDMAIC forem executadas na ferramenta Jira. Essas informações incluem as análises de causa realizadas, as ações de melhoria, os resultados obtidos e as lições aprendidas durante o MiniDMAIC.

Até o momento, já foram realizadas várias ações MiniDMAIC em diversos projetos de software da organização, apresentando ótimos resultados. O tempo de execução dos MiniDMAICs nos projetos levou em média de 4 a 6 semanas, executado por uma equipe com uma média de 3 a 8 pessoas. Participaram da execução dos projetos os seguintes papéis: líder Green Belt disponibilizado pela equipe de qualidade da 
organização, coordenador do projeto, líder de requisitos, analista de teste e desenvolvedor.

As principais ferramentas que têm sido utilizadas para a análise de causas são o Diagrama de Ishikawa e sessões de Brainstorming com as equipes do projeto, para levantamento das potenciais causas do problema, Gráfico de Pareto e métodos de análise e tomada de decisão, para classificação e priorização das causas a serem atacadas e das ações a serem implementadas. A Tabela 9 descreve algumas dessas ações.

Tabela 9. Resultados dos MiniDMAICs executados em projetos de desenvolvimento de software

\begin{tabular}{|c|c|c|c|}
\hline Problemas & Causas Priorizadas & Ações Tomadas & Resultados Obtidos \\
\hline \begin{tabular}{l|} 
MiniDMAIC 1 - \\
Produtividade do \\
projeto muito baixa \\
para atingir os \\
limites de \\
especificação da \\
organização
\end{tabular} & $\begin{array}{l}\text { - Estimativas muito } \\
\text { otimistas } \\
\text { - Falta de experiência } \\
\text { em algumas tecnologias } \\
\text { utilizadas no projeto } \\
\text { - Problemas na } \\
\text { comunicação interna }\end{array}$ & $\begin{array}{l}\text { - Detalhar mais as estimativas, para tentar } \\
\text { avaliar melhor os impactos } \\
\text { - Fazer a implementação de uma parte das } \\
\text { soluções técnicas para ter uma idéia melhor } \\
\text { das dificuldades } \\
\text { - Medir refactoring em outros UCs e incluir } \\
\text { como estimativa bottom-up } \\
\text { - Incluir margem de erro nas estimativas } \\
\text { bottom-up } \\
\text { - Avaliar o impacto antes da adoção de } \\
\text { novas tecnologias e alocar horas para auto- } \\
\text { estudo quando uma nova tecnologia for } \\
\text { adotada } \\
\text { - Realizar reuniões técnicas mensais, para } \\
\text { difundir conhecimento } \\
\text { - Utilizar um wiki para documentar } \\
\text { informações do projeto } \\
\text { - Registrar pendências na ferramenta de } \\
\text { controle de problemas } \\
\text { - Enviar atas de discussões técnicas com o } \\
\text { cliente para a lista do projeto } \\
\text { - Enviar para o cliente, por email, atas com } \\
\text { o resultado de acordos e conversas } \\
\text { - Cobrar aprovação dos casos usos }\end{array}$ & \begin{tabular}{|l} 
Produtividade \\
melhorou $27 \%$ no \\
projeto.
\end{tabular} \\
\hline \begin{tabular}{|l|} 
MiniDMAIC 2 - \\
Alto número de \\
defeitos em um dos \\
projetos da \\
organização
\end{tabular} & $\begin{array}{l}\text { - Não realização de pré- } \\
\text { teste } \\
\text { - Paralelismo entre } \\
\text { A\&P e codificação } \\
\text { - Testes unitários não } \\
\text { são eficazes } \\
\text { - Correções da revisão } \\
\text { de código feitas após a } \\
\text { liberação para testes }\end{array}$ & $\begin{array}{l}\text { - Planejar o pré-teste nos cronogramas das } \\
\text { iterações seguintes } \\
\text { - Incluir folga no planejamento para evitar } \\
\text { paralelismo de A\&P e codificação } \\
\text { - Alocar toda a equipe na elaboração de } \\
\text { testes unitários } \\
\text { - Só autorizar liberação para testes após a } \\
\text { correção dos problemas de Revisão Técnica } \\
\text { de código }\end{array}$ & $\begin{array}{l}\text { Diminuição da } \\
\text { densidade de defeitos } \\
\text { em } 96 \% \text {. }\end{array}$ \\
\hline
\end{tabular}




\begin{tabular}{|c|c|c|c|}
\hline $\begin{array}{l}\text { MiniDMAIC } 3 \text { - } \\
\text { Baixa } \\
\text { produtividade de } \\
\text { engenharia do } \\
\text { projeto }\end{array}$ & $\begin{array}{l}\text { - Complexidade do } \\
\text { projeto, que envolve } \\
\text { duas plataformas bem } \\
\text { diferentes: .NET e Java } \\
\text { - Pouca experiência da } \\
\text { equipe na tecnologia } \\
\text {.NET } \\
\text { - Deficiências no } \\
\text { Sistema Operacional } \\
\text { atual, exigindo vários } \\
\text { refactorings para fazer a } \\
\text { aplicação executar de } \\
\text { maneira aceitável em } \\
\text { relação a desempenho e } \\
\text { memória } \\
\text { - Com a utilização de } \\
\text { tecnologias pouco } \\
\text { maduras, ocorreram } \\
\text { problemas ainda não } \\
\text { identificados, onde foi } \\
\text { gasto parte do esforço } \\
\text { de codificação } \\
\text { pesquisando soluções } \\
\text { para os problemas e } \\
\text { para implementar } \\
\text { soluções alternativas }\end{array}$ & \begin{tabular}{|l|} 
\\
\\
- Realização de treinamentos técnicos \\
- Aumentar o acompanhamento diário do \\
Líder Técnico \\
- Implementar ações de motivação da equipe \\
\end{tabular} & $\begin{array}{l}\text { Produtividade de } \\
\text { Engenharia melhorada } \\
\text { em } 29 \% .\end{array}$ \\
\hline
\end{tabular}

\section{Considerações Finais e Limitações}

Por décadas, as organizações têm utilizado uma infinidade de ferramentas e metodologias diferentes para o tratamento dos problemas, todas elas com diferentes graus de eficácia. No entanto, nenhuma delas visa tratar problemas relacionados especificamente a projetos de desenvolvimento de software. A metodologia MiniDMAIC, proposta neste trabalho, tem o intuito de suprir tal necessidade.

Além disso, espera-se que a utilização do MiniDMAIC possa vir a tornar a implantação da área de processo de Análise e Resolução de Causas do CMMI menos custosa e menos complexa, tornando viável a sua adoção no âmbito dos projetos de desenvolvimento de software.

Assim, pode-se auxiliar as empresas a alcançar níveis de maturidade mais elevados, aumentando a satisfação de seus clientes e reduzindo variações de processos em sua busca pela excelência operacional.

A utilização do MiniDMAIC em projetos reais tem apresentado resultados animadores. Como principal fator para o sucesso dessas ações, temos a busca pela causa-raiz dos problemas.

A utilização de ferramentas de análise de causa, bem como de ferramentas estatísticas implementadas como parte do nível 4 do CMMI, permitem uma análise mais consistente dos problemas e permitem que suas causas reais, e não apenas seus 
sintomas, sejam resolvidas. Assim, o problema é eliminado e não volta a ocorrer, trazendo resultados mais duradouros.

\subsection{Limitações do Trabalho}

O MiniDMAIC foi elaborado no contexto de organizações que possuem pelo menos o nível 3 de maturidade do CMMI. Isso não impede que ele seja aplicado em organizações que não tenham esse nível de maturidade. Porém, alguns passos desta metodologia podem não ter o resultado esperado, devendo ser adaptados para a realidade da organização.

Caso a organização possua o nível 4 do CMMI, as ações MiniDMAIC terão resultados ainda melhores, uma vez que diversos parâmetros para medir os resultados dos projetos já estarão definidos, bem como a utilização de ferramentas de análise estatística já será prática comum na organização.

Além disso, a sua utilização não vem a atender toda a PA de Análise e Resolução de Causas, uma vez que não é intenção do MiniDMAIC tratar os problemas organizacionais. Para estes casos, é recomendado o uso do DMAIC.

\section{Referências}

Banas Qualidade. (2007) "Melhoria contínua - Soluções de Problemas”, Quality News. São Paulo. Disponível em <http://www.estatbrasil.com.br/PgQtN20030003.htm>. Acesso em: 22 fev, 2007.

Blauth, Regis. (2003) "Seis Sigma: uma estratégia para melhorar resultados", Revista FAE Business, $n^{\circ} 5$.

Chrissis, Mary B.; Konrad, Mike; Shrum, Sandy. (2006) "CMMI: Guidelines for Process Integration and Product Improvement", $2^{\text {nd }}$ edition, Boston, Addison Wesley.

Harry D., Schroeder, R. (2000) Six Sigma: the breakthrough management strategy revolutionizing the World's Top Corporation. New York: Doubleday.

Ishikawa, K. (1998) Controle de Qualidade Total - À Maneira Japonesa. Rio de Janeiro,

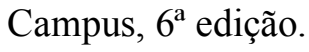

Juran, J. M. (1991) Controle de Qualidade Handbook. J. M. Juran, Frank M. Gryna São Paulo - Makron, McGraw-Hill.

Kulpa, Margaret K.; Johnson, Kent A. (2003) "Interpreting the CMMI: a process improvent approach" Florida, Auerbach.

Pande, S. (2001) "Estratégia Six Sigma: como a GE, a Motorola e outras grandes empresas estão aguçando seu desempenho". Rio de Janeiro, Qualitymark.

Rath and Strong. (2005) “Six Sigma/DMAIC Road Map", 2 nd edition.

Rotondaro, G. R; Ramos, A. W.; Ribeiro, C. O.; Miyake, D. I.; Nakano, D.; Laurindo, F. J. B; Ho, L. L.; Carvalho, M. M.; Braz, A. A.; Balestrassi, P. P. (2002) "Seis Sigma: Estratégia Gerencial para Melhoria de Processos, Produtos e Serviços", São Paulo, Atlas.

Smith, B.; Adams, E. (2000) "LeanSigma: advanced quality", Proc. 54 $4^{\text {th }}$ Annual Quality Congress of the American Society for Quality, Indianapolis, Indiana. 
Tayntor, Christine B. (2003) "Six Sigma Software Development”, Flórida, Auerbach.

Watson, G. H. (2001) "Cycles of learning: observations of Jack Welch", ASQ Publication. 